\title{
Allocation of scarce psychotherapeutic resources: two landmark documents
}

\author{
Jeremy Holmes
}

If psychiatry is the Cinderella of medicine, psychotherapy in the National Health Service (NHS) is often the Cinderella of the Cinderellas underfunded, undervalued, and underused. The recently published National Health Service Executive (NHSE) document The Future of Psychotherapy Services (Department of Health, 1996) is a determined and impressive attempt to document and to redress this. It starts from the position that the psychotherapies are effective treatments for a wide range of psychiatric conditions (Roth \& Fonagy, 1996). The use of cognitive therapy and interpersonal therapy in depression (which in no trial so far has been shown to be less effective than antidepressants), family therapy in eating disorders, and dynamic therapy in chronic functional bowel disorder are but three well-established examples of psychotherapeutic effectiveness, based on welldesigned random controlled trials (Aveline \& Shapiro, 1995).

The main recommendations of the report are that psychotherapy services should be comprehensive, coordinated, user-friendly, safe, clinically effective and cost effective. In an attempt to focus on the latter two requirements, a parallel volume, What Works for Whom (Roth \& Fonagy. 1996), also commissioned by the NHSE, provides a comprehensive Which-type guide to the evidence for the effectiveness of different types of psychotherapy in the main psychiatric disorders.

As a background to the NHSE document its authors surveyed how, and to what extent, psychotherapy is available as a treatment with trusts. The results showed a wide variation between districts, although the majority were by most standards under-resourced. Another finding was the huge variety of services and individuals described as delivering psychotherapy. Thus a district psychotherapy service may contain a diverse group of practitioners including: qualified or unqualified counsellors employed in general practice; community mental health workers such as community psychiatric nurses who may have had specific NHS-based training as nurse behaviour therapists, or private training in one of the humanistic therapies; a district psychology service comprising eclectically trained consultant psychologists often specialising in cognitive behaviour therapy: a child psychiatry service with expertise in family therapy; and consultant psychotherapists in a psychotherapy department, often offering predominantly analytic psychotherapy.

The main dimensions of this diversity are: (a) modality of therapy (e.g. analytic, cognitivebehavioural, family), (b) length and depth of training; and (c) delivery (e.g. in general practice, as part of a 'package' of treatment in secondary care, or in a specialised psychological treatment unit). In an attempt to rationalise this diversity, the NHSE document (Department of Health, 1996) introduces a new classification in which psychotherapy is divided into three types. Type A psychotherapy is combined with other treatments for specific psychiatric disorders: for example cognitive behaviour therapy or family therapy in schizophrenia, or interpersonal therapy in eating disorders. Here psychotherapy follows from the diagnosis. The patient is seen as having a diversity of needs, some of which are amenable to psychotherapy. Type B psychotherapy is patient-centred, eclectic psychotherapy in which the treatment is tailored to the needs of the particular individual, who might be suffering from depression, relationship difficulties or a personality disorder and may require a mixture of cognitive, behavioural and analytic approaches at different stages of therapy. Type $\mathrm{C}$ therapies are model-based formal psychotherapies in one of the distinct modalities: analytic, cognitive etc. The report implies that Type A is likely to be delivered by nurses as part of a general psychiatric service. Type B by psychologists working in psychology departments, and Type $\mathrm{C}$ under the direction of consultant psychotherapists in psychotherapy departments.

What are the implications of this for local mental health services? The report endorses the move towards integrated multi-disciplinary psychological treatment services, in which 
therapists of differing orientation collaborate to offer the full range of therapies. The psychological treatment service provides a focus for planning, audit and research which are the essential prerequisites of a coordinated and comprehensive service. It argues that there is no place in contemporary psychiatry for the ivory towerism and mutual suspicion which has hitherto isolated some psychotherapy services from the mainstream of psychiatry, and some of the different components of a comprehensive service (notably psychology and medical psychotherapy) from one another. It advocates bringing psychotherapy within the orbit of the Care Programme Approach, so that the psychotherapeutic needs of each patient referred to secondary services are systematically considered.

This raises one of the besetting dilemmas of all psychological treatment services. With the move into the community, there is a tension between the needs of the community teams for psychotherapeutic input (Type A), and the necessity for a specialist psychotherapy service to concentrate on their primary tasks of developing the expertise needed to treat difficult cases (Types B and C); and between the need for a critical mass of psychotherapy practitioners, and offering training to the mental health unit generally. Should psychotherapists base themselves in the community teams, offering supervision and backup to these secondary services, or should they concentrate on developing a tertiary centre of excellence, at some remove from the front line of mental health work?

Clearly some compromise will be worked out according to local conditions and need. Whatever the arrangements, psychotherapy assessment by a senior practitioner is an essential function of a psychotherapy service. Assessment has many functions, of which triage is perhaps the most important. Scarce specialist resources must be concentrated on those most in need, and who are most likely to benefit. The issue was summarised in Pauls's (1967) well-known formula: 'What treatment delivered by whom is most effective for this individual, with that specific problem, and under which set of circumstances?' Patients with defined problems of short duration which are not producing major functional impairment can be treated by one of the brief psychotherapies, often in general practice, with secondary services offering support and supervision. At the other end of the spectrum, those with intractable problems unlikely to benefit from intensive psychotherapy should, perhaps after a trial of formal therapy, be offered low-intensity supportive psychotherapy (Holmes, 1995) either individually or in groups. The remainder, whom the report shows are severely ill by any medical or psychiatric criteria and are certainly not the 'worried well', deserve intensive treatment by well trained and supervised specialist staff. The most ill of all may need specialised in-patient or day patient psychotherapy units if their complex needs are effectively to be met.

The review and the research survey are landmark documents in the evolution of psychotherapy within the NHS, providing a programme for service development, audit, and research for at least a decade. It is now the urgent task of purchasers, trusts, and practitioners to translate that programme into action.

\section{References}

AVEune. M. \& ShaPIRo. D. (1995) Research Foundations for Psychotherapy Practice. Chichester: Wiley.

DEPARTMENT OF HEALTH (1996) NHS Psychotherapy Services in England: Review of Strategic Policy. London: HMSO.

HOLMES, J. (1995) Supportive psychotherapy: the search for positive meanings. British Joumal of Psychiatry. 167. $439-445$.

PAUL. G. L. (1967) Strategy of outcome research in psychotherapy. Journal of Counselling Psychology. 31. 109-118.

RoTH, A. \& FonAGY, P. (1996) What Works for Whom? A Critical Review of Psychotherapy Research. New York: Gutlford Press.

Jeremy Holmes, Consultant Psychiatrist/ Psychotherapist, North Devon District Hospital, Barnstaple, Devon EX31 4JB; Fax 01271311523 ; e-mail: aaJ64@dial.pipex.com 